American J. of Engineering and Applied Sciences 3 (3): 509-515, 2010

ISSN 1941-7020

(C) 2010 Science Publications

\title{
The Effects of Sense of Progression and Cognitive Distance on Path Choice and Walking Behavior While Aiming To Reach Destination
}

\author{
Mohammad Paydar and Samira Ramezani \\ Department of Architecture and Urban Design, Faculty of Built Environment, \\ University Technology Malaysia, 81310, UTM, Johor, Malaysia
}

\begin{abstract}
Problem statement: Studies focused on walking behavior have indicated the relationship of different factors of built environment with walking to reach destination and walking for recreation. Furthermore, according to literature on path choice behavior, there is a relationship between walking behavior and path choice of pedestrians. Empirical studies on path choice behavior have also shown that the effects of different environmental variables on path choice vary with the purpose of the trip; whether recreational purposes or work-related walking trips. However there is a necessity to theoretically understand why consideration of purpose of the trip is important in studies of walking as well as path choice behavior. Furthermore, since reaching the destination is the most important travel purpose in daily activities, the environmental variables related to walking to reach the destination as well as path choice when aiming to reach destination need special consideration, if the goal is to encourage walking in daily basis. Approach: This study, therefore, relied on literature review to find answer to the research questions. Two concepts of instrumental and divertive behavior were used to answer the first research question. Research proceeded with making an effort to extract and introduce the main environmental variables related to walking and path choice of pedestrians when aiming to reach the destination. Results: Based on the definition of instrumental and divertive behavior, the necessity of consideration of purpose of the trip and its effects on environmental variables affecting walking as well as path choice behavior were theoretically verified. Moreover, two factors of cognitive distance and sense of progression were found to be the most important factors affecting walking and path choice behavior while aiming to reach destination. The physical features contributing to creation of sense of progression were also extracted and introduced. It is also suggested that sense of progression affects walking and path choice behavior through affecting cognitive distance and generating positive affective responses. Conclusion: It is suggested that future empirical studies be conducted to support the hypothesized relationships extracted and introduced in this study. Such studies would contribute to planning and design of urban spaces which would encourage walking in a daily basis.
\end{abstract}

Key words: Built environment, physical features, environmental factors, instrumental behavior, divertive behavior

\section{INTRODUCTION}

Walking is an activity almost everyone engages in. It offers a wide range of benefits to both individuals and society (Gehl, 1987). From a transportation standpoint, walking leads to less vehicular travel and thus less traffic, air pollution and other environmental impacts. From a public health standpoint, walking means increased physical activity and thus improved health and reduced healthcare costs. A growing number of empirical studies from the above-mentioned standpoints have contributed to the debate on the relationship between built environment and walking behavior (Cervero and Radisch, 1996; Black et al., 2001; Greenwald and Boarnet, 2001; Handy and Clifton, 2001; Ball et al., 2001). Put differently, those studies provide an evidence for a correlation between built environment and walking. However, in recent years, the issue concerned by a number of researchers is that the impact of built environment on walking behavior may depend on the purpose of the trip; whether walking to reach the destination or walking for recreational purposes (Cao et al., 2006). In regard to this gap, a new stream of researches surveyed the influences of various

Corresponding Author: Mohammad Paydar, Department of Architecture and Urban Design, Faculty of Built Environment, University Technology Malaysia, 81310, UTM, Johor, Malaysia 
built environmental variables such as aesthetics and distance on walking behavior on the basis of purpose of the trip (Pikora et al., 2003; Owen et al., 2004; Suminski et al., 2005).

On the other hand, Path choice behavior or, in other words, how people react and choose their favorite path in decision points such as urban nodes and junctions is correlated with walking behavior as there might be some previous walking experiences in the path which would affect opting for it. Pedestrian behavior models in urban spaces can be applied to show the relationship between walking behavior and path choice behavior (Hoogendoorn and Bovy, 2004; Kitazawa and Batty, 2004).

Since walking is one of the main modes of transportation especially along the short distance between origin and destination, a number of pedestrian behavior models in urban settings have considered walking as a mode of travel for pedestrians especially in the small-scale environments such as streets. Utility Maximization Theory is one of the main theories applied in travel behavior studies. Several models based on the utility maximization theory have been suggested and tested in order to understand and predict the behavior of pedestrians in the urban setting (Hoogendoorn and Bovy, 2004; Kitazawa and Batty, 2004). The main theoretical assumption in Utility Maximization Theory is that pedestrians make a subjective rational choice between alternatives. In other words, in the models which are formed based on the concept of utility maximization theory, the focus is on the choice-based approaches, in which observed behavior is typically viewed as a manifestation of people's preferences and individuals are assumed to choose the alternative that maximizes their utility (Hoogendoorn and Bovy, 2005).

Figure 1 show the concept of one of those models which are based on the concept of utility maximization theory. Based on this model, the choice could be distinguished at the following three levels (Hoogendoorn and Bovy, 2004; Kitazawa and Batty, 2004):

- Strategic level: Destination choice

- Tactical level: Route choice to reach the destination

- Operational level: Walk ability of the path and walking behavior
In this hierarchy, expected utilities at lower levels influence choices at higher levels and Choices at higher levels condition choice sets at lower levels (Hoogendoorn and Bovy, 2004). Therefore, the environmental factors which affect walking behavior could be taken into account in the path choice behavior and path choice behavior is influenced by the factors affecting walk ability of the path and walking behavior.

The tactical behavioral model (path choice behavior) is influenced by both external factors (e.g., presence of obstacles, stimulation of the environment) and internal (or personal) factors (age, gender, attitudes of the pedestrian) (Hoogendoorn and Bovy, 2004; 2005). Empirical studies indicate that, the most important external factor is the shortest path which can be interpreted as shortest distance and least time of the trip between origin and destination (Hill, 1982; Golledge, 1995). A study found that direction is the most usual criteria for choosing a particular path. The directness of the route is related not only to length of the route but also to its complexity (in terms of direction change) (Hill, 1982). Another study found that in addition to shortest distance and least time there are some other criteria for path choice such as paths with fewer turns and most aesthetically appealing path (Golledge, 1995). One study emphasized on Pleasantness along the path as an important criterion for path choice (Bovy and Stern, 1990). Other factors considered important in route choice behavior are habit, number of crossings, pollution and noise levels, safety and shelter from poor weather conditions and stimulation of the environment (Hoogendoorn and Bovy, 2004).

The extent to which abovementioned route attributes play a substantial role in route choice behavior depends to a large extent on trip purpose (Bovy and Stern, 1990). Scenery is very important for recreational trips, but it plays no role in work-related walking trips (Bovy and Stern, 1990). Therefore, the effects of environmental variables on path choice behavior depend on the purpose of the trip, consisting of recreational trips and work-related walking trips. As mentioned above, the Necessity of consideration of purpose of the trip was also emphasized in the literature on walking behavior.
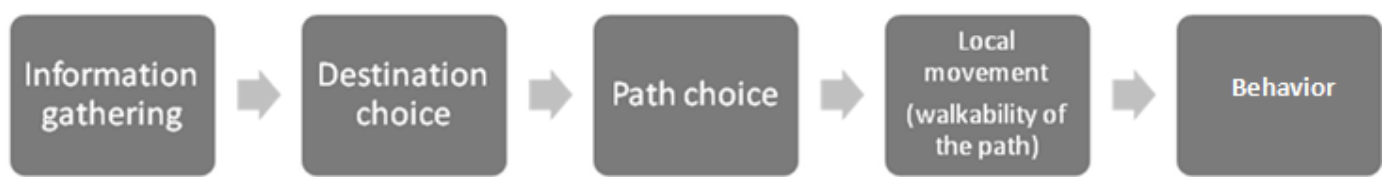

Fig. 1: Concept of pedestrian behavioral model suggested by Kitazawa and Batty (2004) 
The question that arises here is that how the necessity to consider the relationship between environmental variables and walking behavior as well as path choice behavior based on the purpose of the trip, consisting of recreational purposes and reaching to the destination, can be theoretically understood.

Furthermore, as most people transport in the city between different points, especially in work-related walking, walking to reach destination forms the highest rate of walking conducted in daily activities. Therefore, consideration of the important environmental variables correlatives with walking to reach the destination as well as path choice to reach the destination seems necessary in the pedestrian behavior studies. This study, therefore, scrutinizes the most important environmental variables that affect walking and path choice behavior with an especial emphasis on walking trips to reach the destination.

\section{MATERIALS AND METHODS}

The method used in this study is analysis of data gathered through review of literature. By applying the two concepts of Instrumental and diversive behavior, this study makes an effort to answer the first raised question so as to theoretically understand the necessity of considering the environmental factors on the basis of purpose of the walking trips. This study proceeds with reviewing the related literature to extract the most important environmental factors correlated with path choice and walking to reach destination. The reasons for importance of those factors in path choice and walking to reach destination and the relationship between those factors are discussed as well.

\section{RESULTS}

Instrumental versus divertive behavior: Review of literature suggested that the two concepts of instrumental and divertive behavior could help in understanding of the effects of different environmental variables on walking behavior and path choice behavior based on the purpose of the walking trips. These two types of behavior were defined by Heath (1988) in Nasar's book on environmental aesthetics. According to him, Instrumental objectives are explained as motivations linked to completion of a particular task, such as way finding, finding a grocery store or a metro station.

Diversive behavior, however, is linked to exploration; the behavior of the tourist, window shopper and stroller. The main goal of this type of behavior is to simply experience or pursue the activity.
Attention to the environment is very different from that of an instrumental mindset, since such diverted individuals are paying more attention to the space as a whole. Information acquired in an exploratory state is different from a task-oriented state. It allows more attention, slower movement and more time, all of which allow for collection of more information about the environment (Zacharias, 2001). To exemplify, on instrumental behavior or task-oriented behavior one may focus on a large landmark and build information around it, whereas during an exploration of a space one may focus on a schematic element in space, such as the color of the walls or the number of the people in the space (Seto, 2008).

The importance of distance and cognitive distance in path choice and walking behavior to reach destination: Findings of studies surveying the effects of environmental factors on walking, by segregating it into walking to reach destination and walking for recreation, indicate that distance to destination is one of the most effective factors on walking to reach destination (Suminski et al., 2005; Owen et al., 2004; Pikora et al., 2003). Knowledge of distance in the environment affects the decision to stay or go, the decision of where to go and the decision of which route to take (Cadwallader, 1976). The literature on path choice behavior also indicate time and distance to destination to be the most important factors pedestrians take into account while choosing the path (Hill, 1982; Golledge, 1995). However, an understanding of the perception and cognition of distance is fundamental to the prediction and explanation of spatial behavior (Montello, 1997).

Sense of progression along the path and its importance for walking behavior as well as path choice behavior toward destination: The instrumental behavior which was discussed to have an association with walking to get to destination emphasizes that the quality of path should help in accomplishing the task (the task of reaching destination in this case). Sense of progression is a factor along the path which offers people a sense of approaching to destination thus helping them do the task of getting to their destinations.

Sense of progression can be explained in this way that the path is perceived, in fact, as a thing which goes towards something. The path should support this perceptually so that it is given a sense of progression while the opposite directions are unlikely (Lynch, 1960). Lynch (1960) indicates that sense of progression can be generated by strong termini especially when it is visible along the route, a gradient 
or a directional differentiation. Therefore sense of progression is a quality of the path which not only makes people realize their position along the path but also helps them have a fine navigation towards their destination. Thus proper navigation toward destination is correlated with sense of progression along the walkways.

\section{DISCUSSION}

By comparing the instrumental and divertive behavior with walking behavior on the basis of the two different purposes of the trip, whether walking to reach destination or for recreational purposes, it could be interpreted that the instrumental behavior is a kind of behavior related to work-related walking trip towards defined destination and path choice of those who want to reach their destination while divertive behavior is coordinated with walking for recreation and path choice of those with recreational purposes. Therefore, any environmental factors helping people accomplish their task (here the task of reaching destination) can be important for walking to reach destination and consequently could be taken into account for path choice. Cognitive distance and sense of progression are two factors, extracted from literature, with such characteristics.

It was mentioned that distance to destination is an important factor for walking to reach the destination as well as path choice behavior of those who want to reach the destination but how distance or time is measured by pedestrians is worthy of reflection. Pedestrians use their mental measurement of the path based on their previous walking experiences. Mental measurements of path lengths are often not accurate and are more often subjectively derived through other qualitative measurements. Distance in spatial representation is not encoded in terms of metric distances but in a schematic measure such as cognitive storage space, cognitive time or cognitive effort (Jansen-Osman and Berendt, 2002). Among the environmental factors, having been indicated to have a correlation with cognitive distance, the number of junctions and landmarks along the route can be referred to, increasing of which contributes to increasing the cognitive distance along the path (Sadalla and Staplin, 1980). Visibility of destination is another factor which affects the cognitive distance and decreases it (Nasar et al., 1985). Therefore, cognitive distance, rather than actual distance of the trip, could be taken into account as a factor affecting path choice as well as walking behavior toward destination and presence of some physical elements and features along the path and density of them affect determination of the cognitive distance of the trip by pedestrians.
Literature suggested that there is a strong relationship between sense of progression and well navigation along the path. Navigation is a sequence of path decisions made and executed within an urban space (Stern and Portugali, 1999). Survey knowledge and route knowledge are two types of spatial representation knowledge and are formulated during navigation (Seto, 2008). Both of these forms of knowledge grow as one becomes more familiar with the urban environment (Seto, 2008). Route knowledge refers to one's ability to navigate a formerly learned path within an environment effectively (Bliss et al., 1997). Route knowledge is composed of two main components: (1) landmarks, which are linked to orientation knowledge and (2) path intersections, which are points of reference (Richter et al., 2004). On the other hand, Survey knowledge is often defined as an integrated understanding of an environment and the way spaces are related to each other, which includes knowledge of spatial element interrelationship (McDonald and Pellegrino, 1993). Therefore, survey knowledge emphasizes on the visual and perceptual connectivity of the elements such as landmarks and intersections which contribute to understanding the connectivity of different parts and making a whole image of the routes easily.

Based on the relationship between well navigation and sense of progression and also the definition of sense of progression, the following factors are suggested to have an effect on the sense of progression along the path:

- Presence of junctions (intersections) and landmarks along the path

- Visual connectivity and visibility of landmarks along the path which work as an aid for orientation

- Location of landmarks and intersections in relation to the destination points in order to create what Lynch (1960) refers to as a gradient or a directional differentiation

Sense of progression was defined as an important quality of the path especially for walking to reach the destination. However, presence of sense of progression along the walkways is not only an important factor in walking behavior of those who want to reach their destination, but also in their path choice behavior. A study surveying the criteria for path choice between a presented origin and destination found that choice of routes varied depending on direction of travel. In dead, the path that respondents chose when travelling from origin to destination was different from that chosen when returning to origin. It was discussed that one 
interpretation of this route choice behavior is that respondents chose a route that took them away from origin point as soon as possible when leaving the origin point and took them close to destination point as quickly as possible when approaching their destination points (Golledge, 1995). In fact such a directional differentiation in the perception of the respondents was caused by the location of the elements along the path in relation to origin and destination points which is one of the factors that contribute to generating sense of progression along the path. In fact, the difference in special configuration of environment in different directions leads to different rate of sense of progression perceived along the paths and affects path choice behavior of pedestrians. Figure 2 shows the theoretical framework of this study.

The way sense of progression affects walking as well as path choice behavior towards destination: According to literature, sense of progression could affect walking behavior as well as path choice behavior to reach the destinations through.

Affecting cognitive distance of the trip: According to the results of this study, there seems to be a relationship between sense of progression and cognitive distance along the path. As mentioned before, the studies on cognitive distance indicate a correlation between the number of elements such as junctions and landmarks with cognitive distance (Sadalla and Staplin, 1980). These studies also indicate the relationship between visibility of destination and cognitive distance (Nasar et al., 1985). Visibility of destination is a factor contributing to visual connectivity of elements along the path. On the other hand, these physical features consisting of visibility of destination and number of nodes and junction along the path could be taken into account as the components generating sense of progression. Therefore, there is a relationship between generation of sense of progression and cognitive distance along the path. In other words, it could be assumed that sense of progression affects path choice behavior as well as walking behavior to reach the destination through affecting cognitive distance of the trip.

Generating positive affective responses such as stimulating, pleasant and comfortable along the path: According to the results of this study, it seems that generating sense of progression along the path is companion with creation of some affective responses. It was pointed out that sense of progression makes pedestrians feel that they know their location along the path and such a feeling may contribute to generating sense of comfort.

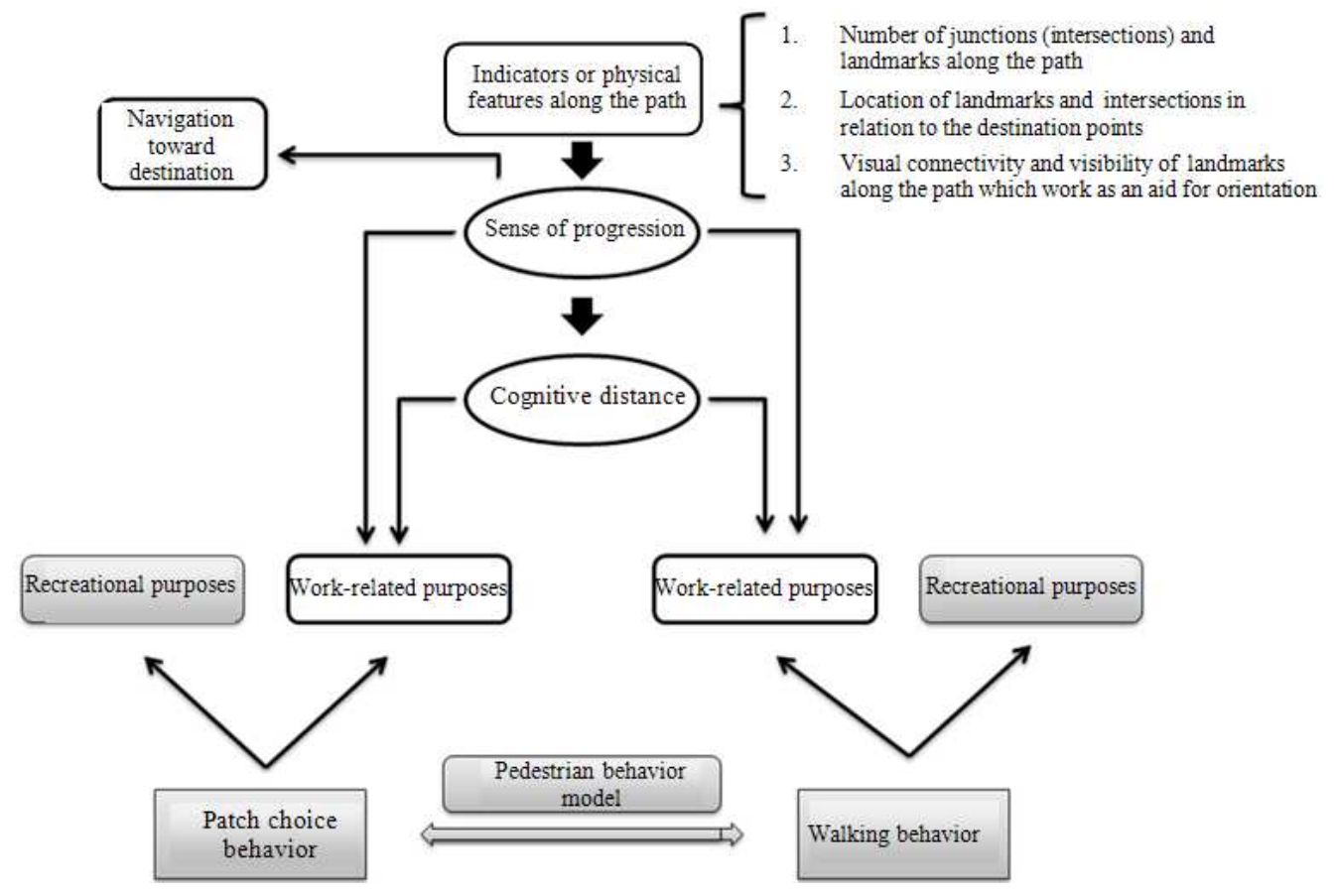

Fig. 2: Theoretical framework of the current study 
Am. J. Engg. \& Applied Sci., 3 (3): 509-515, 2010

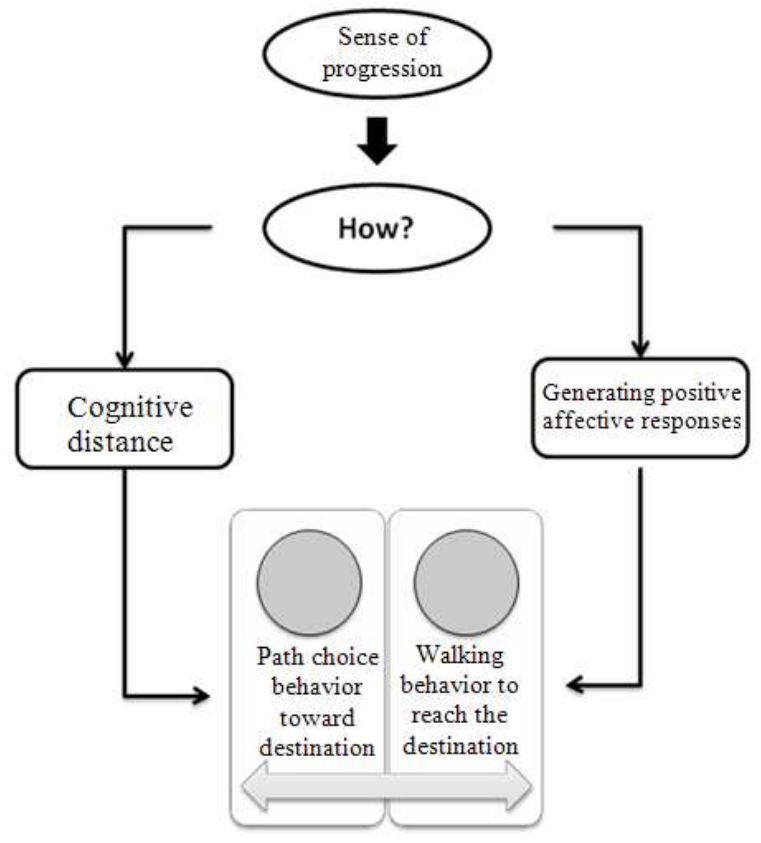

Fig. 3: Framework on the way sense of progression affects walking as well as path choice behavior while aiming to reach destination

A study on urban aesthetic experiences found that some aesthetic factors such as visibility of landmarks along the path, which is one of the components of sense of progression, contribute to generation of intense aesthetic affections. These aesthetic affections are mostly defined as stimulating and interesting (Isaacs, 2000). Therefore, this is assumed that sense of progression through its affect on positive affective responses such as sense of comfort, stimulation and interest along the path could affects walking and path choice behavior of pedestrians who want to reach their destination. Figure 3 shows the above mentioned relationships.

\section{CONCLUSION}

This study emphasized that the effects of environmental factors on path choice behavior as well as walking behavior along the path depend on the purpose of the trip; whether recreational or workrelated walking trips (walking to reach the destination). Based on the related literature, two concepts of diversive and instrumental behavior were reviewed to theoretically define why effects of environmental variables on walking behavior as well as path choice behavior depend on the purpose of the trip. Furthermore, two factors of sense of progression and cognitive distance were extracted as the important factors affecting walking behavior and path choice of pedestrians who want to reach their destination. Based on the relationship between the definition of sense of progression and navigation of the pedestrians along the path and applying the concepts of survey knowledge and path knowledge a number of physical features were suggested as the features contributing to generation of sense of progression along the path. It was also suggested and discussed that sense of progression affects walking and path choice behavior through affecting cognitive distance and generating positive affective responses.

It is suggested that future empirical studies be conducted to support the hypothesized relationships extracted and introduced in this study. The effects of the two factors of cognitive distance and sense of progression on path choice and walking behavior of those who want to reach their destination need to be supported by future empirical studies. The relationship between related physical features extracted from literature and sense of progression and the importance of each in generation of sense of progression, also need to be empirically surveyed. Future research could also be conducted to empirically understand whether sense of progression affects path choice and walking behavior through its affects on cognitive distance or positive affective responses. Such studies would contribute to planning and design of urban spaces which would encourage walking in a daily basis.

\section{REFERENCES}

Ball, K., A. Bauman, E. Leslie and N. Owen, 2001. Perceived environmental aesthetics and convenience and company are associated with walking for exercise among Australian adults. Prevent. Med., 33: 434-440. DOI: 10.1006/pmed.2001.0912

Black, C., A. Collins and M. Snell, 2001. Encouraging walking: The case of journey-to-school trip in compact urban areas. Urban Stud., 38: 1121-1141. DOI: 10.1080/0042098012005168-4

Bliss, J.P., P.D. Tidwell and M.A. Guest, 1997. The effectiveness of Virtual Reality for administering Spatial Navigation Training to Firefighters. Presence: Teleoperat. Virtual Environ. J., 6: 73-86.

Bovy, P.H.L. and E. Stern, 1990. Route Choice: Way finding in Transport Networks. Kluwer Academic Publishers, Dordrecht, ISBN: 0792308123, pp: 309.

Cadwallader, M.T., 1976. Cognitive Distance in Interurban Space. In: Environmental Knowing: Theories, Research and Methods, Moore, G.T. and R.G. Golledge, (Eds.). Dowden, Hutchinson and Ross, Inc., Stroudsburg, Pennsylvania, ISBN: 0879330600, pp: 316-324. 
Cao, X., S.L. Handy and P.L. Mokhtarian, 2006. The influences of the built environment and residential self-selection on pedestrian behavior: Evidence from Austin, TX. Transportation, 33: 1-20. DOI: 10.1007/s11116-005-7027-2

Cervero, R. and C. Radisch, 1996. Travel choices in pedestrian versus automobile oriented neighborhoods. Transport Policy, 3: 127-141. DOI: 10.1016/0967-070X(96)00016-9

Gehl, J., 1987. Life between Buildings: Using Public Space. 5th Edn., The Danish Architectural Press, Copenhagen, ISBN: 8774072838, pp: 202.

Golledge, R.G., 1995. Path Selection and Route Preference in Human Navigation: A Progress Report, Spatial Information Theory a Theoretical Basis for GIS. COSIT, Springer Berlin/Heidelberg, ISBN: 978-3-540-60392-4, pp: 207-222.

Greenwald, M.J. and M.G. Boarnet, 2001. Built environment as determinant of walking behavior: Analyzing nonwork pedestrian travel in Portland, Oregon. Trans. Res. Rec., 1780: 33-41. DOI: 10.3141/1780-05

Handy, S.L. and K.J. Clifton, 2001. Local shopping as a strategy for reducing automobile travel. Transportation, 28: 317-346. DOI: 10.1023/A:1011850618753

Heath, T., 1988. Behavior and Perceptual Aspects of the Aesthetic of Urban Environments. In: Environmental Aesthetics: Theory, Research and Applications, Nasar, J.L. (Ed.). Cambridge University Press, Cambridge, ISBN: 0521429161, pp: 6-10.

Hill, M.R., 1982. Spatial structure and decision-making aspects of pedestrian route selection through an urban environment. Collection for University of Nebraska-Lincoln, Paper AAI8306484. http://digitalcommons.unl.edu/dissertations/AAI8306484

Hoogendoorn, S.P. and P.H.L. Bovy, 2004. Pedestrian Route-choice and activity scheduling theory and models. Trans. Res. Part B: Methodol., 38: 169190. DOI: $10.1016 /$ S0191-2615(03)00007-9

Hoogendoorn, S.P. and P.H.L. Bovy, 2005. Pedestrian travel behavior modeling. Networks Spat. Econ., 5: 507-535. DOI: 10.1007/s11067-005-2629-y

Isaacs, R., 2000. The urban picturesque: An aesthetic experience of urban pedestrian places. J. Urban Des., 5: 145-180. DOI: 10.1080/713683961

Jansen-Osman, P. and B. Berendt, 2002. Distance knowledge using virtual environments. Environ. Behav., 34: 178-193. DOI: 10.1177/0013916502034002002

Kitazawa, K. and M. Batty, 2004. Pedestrian behavior modeling, an application to retail movements using a genetic algorithm. DOI: 10.1.1.113.2036
Lynch, K., 1960. The Image of the City. The MIT Press, pp: 190.

McDonald, T.P. and J.W. Pellegrino, 1993. Psychological Perspectives on Spatial Cognition. In: Behavior and Environment: Psychological and Geographical Approaches, Garling, T. and R.G. Golledge, (Eds.). Elsevier, N. Holland, pp: 47-82.

Montello, D.R., 1997. The Perception and Cognition of Environmental Distance: Direct Sources of Information. In: Spatial Information Theory: A Theoretical Basis for GIS, Hirtle, S.C. and A.U. Frank, (Eds.). Berlin, Springer, Germany, ISBN: 978-3-540-63623-6, pp: 297-311.

Nasar, J.L., H. Valencia, Z.A. Omar, S. Chueh and J. Hwang, 1985. Out of sight further from mind, Destination visibility and distance perception. Environ. Behav., 17: 627-639. DOI: 10.1177/0013916585175004

Owen, N., N. Humpel, E. Leslie, A. Bauman and J. Sallis, 2004. Understanding environmental influences on walking review and research agenda. Am. J. Prev. Med., 27: 67-76. PMID: 15212778

Pikora, T., G. Corti, F. Bull, K. Jamrozik and R.J. Donovan, 2003. Developing a framework for assessment of the environmental determinants of walking and cycling. Soc. Sci. Med., 56: 1693-1703. DOI: 10.1016/S0277-9536(02)00163-6

Richter, K.F., A. Klippel and C. Freksa, 2004. Shortest, fastest-but what next? A different approach to route directions.

http://www.crcsi.com.au/uploads/resources/RESO URCES_66.pdf

Sadalla, E.K. and L.J. Staplin, 1980. The perception of traversed distance: Intersections. Environ. Behav., 12: 167-182. DOI: $10.1177 / 0013916580122003$

Seto, D., 2008. Are Pedestrian Path Choices during Exploration Contingent on Measures of Shape Complexity and Visual Content of the Environment, Master thesis, Concordia University, Montreal, Quebec, Canada, ISBN: 9780494408360.

Stern, E. and J. Portugali, 1999. Environmental Cognition and Decision Making in Urban Navigation. In: Way finding Behavior: Cognitive Mapping and Other Spatial Processor, Gollege, G. (Ed.). The Johns Hopkins University Press, Baltimore, ISBN: 080185993X, pp: 99-121.

Suminski, R., S. Walker, C. Poston, R. Petosa, E. Stevens and L.M. Katzenmoyer, 2005. Features of the neighborhood environment and walking by US adults. Am. J. Prevent. Med., 28: 149-155. DOI: 10.1016/j.amepre.2004.09.009

Zacharias, J., 2001. Path choice and visual stimuli: Sign of human activity and architecture. J. Environ. Psychol., 21: 341-352. DOI: 10.1006/jevp.2001.0225 